\title{
Joint Resource Allocation and Spectral Radiometry in Non- Stationary Cognitive Radio Networks
}

\author{
Pouya Derakhshan-Barjoei \\ Assistant Professor, Young Researchers and Elite Club, Department of Telecommunication Engineering, \\ Naein Branch, Islamic Azad University, Isfahan, Iran \\ *Corresponding Author: Pouya Derakhshan-Barjoei, Assistant Professor, Young Researchers and Elite \\ Club, Department of Telecommunication Engineering, Naein Branch, Islamic Azad University, Isfahan, Iran
}

\begin{abstract}
Resource allocation and spectrum sensing are quite important part in cognitive radio networks. In his paper, joint energy sensing or radiometry, the common spectrum sensing algorithm in secondary radio, and distributed power control by beamforming approach in a secondary radio network have been investigated. Power control regulates the transmission power of the secondary users and radiometry indicates the spectrum vacancies. In this paper, we performed a spectrophotometric composition with evolutionary optimization in a cognitive network. We assume primary users are idle or active and therefore secondary users will transmit with maximum power and assigned power, respectively. As a result, we propose an improved performance to solve the constrained nonlinear multi-object optimization and coherent power assignment by beamforming problem based on Particle Swarm Optimization (PSO). A numerical study is performed to show the convergence behavior of the proposed algorithm and the efficiency of the proposed technique.
\end{abstract}

Keywords: Non-stationary cognitive radio, Evolutionary Algorithm, Spectrum sensing, resource allocation.

\section{INTRODUCTION}

The combination of spectral detection with evolution optimization in the cognitive network and trying to increase the output rate and increase the efficiency of the problem is very interesting and important. Spectral radiography is very useful in wireless sensor networks and cognitive radio networks, especially in non-stationary networks, and helps in early detection of interferences. Energy is usually a scarce commodity in wireless ad hoc networks, as users typically operate on batteries, which in many cases are difficult to replace or recharge. In general, cognitive wireless radio network is capable of adapting to the outside existing time varying environment. To avoid harmful interference between different networks to affect users, most spectrum bands are allocated to certain services but worldwide spectrum occupancy measurements show that only portions of the spectrum band are fully used. Moreover, there are large temporal and spatial variations in the spectrum occupancy. When using spectrum sensing, the hidden terminal problem might cause problems when there is an obstacle between the secondary system and the primary transmitter [1,2]. In this condition the secondary user might have good connection to primary receiver but it can not necessarily detect the primary transmitter and its transmission. To overcome this problem, we can use longer sensing period to increase the measurement accuracy but this reduce the available time for transmission, also another method to overcome the problem is to use cooperation. Spectrum 
sensing determines the presence or absence of the primary user, based on a specific detection model. Because of dynamic feature of the environment the transmit power control requires a precise study by employing an intelligent algorithm [3]. While, the population adaptation for genetic algorithm based cognitive radio and bio-inspired algorithm for dynamic resource allocation and parameter adaptation have been studied, in [4,5]. In this paper, we proposed an intelligent method to encounter the challenges of the secondary radio network. We formulate an optimization problem, considering the strict requirement of the protection of licensed users from the interference caused by the unlicensed or secondary users. A challenge with implementing beamforming in ad hoc networks is that the geometry of the network may change dynamically [6,7]. Due to the variation of radio channel characteristics secondary radio networks need to support time varying quality of service requirements. The basic goals of our work are focused on dynamic constrained power allocation, maximizing the transmission capacity by pre and post beamforming technique when primary users are active and secondary users work in an underlay mode or when primary users are idle and secondary users work in an overlay mode. To achieve these goals we employ the algorithm based on particle swarm optimization.

\section{NETWORK MODELING}

We consider a system model where the primary network consists of $N$ primary users (PUs) each having a transceiver system. There is no active communication or cooperative behavior between primary and secondary users. The secondary network has an ad hoc scenario and work in the same frequency band as the primary system. With deployment of $K$ antennas at each secondary transmitter, an efficient transmit beamforming technique is proposed to maximize the sum throughput. The transmit powers of secondary users are limited to a maximum value prescribed by primary users. The system model of our scenario is illustrated in figure 1 . The secondary network included $M$ secondary users so it has an ad hoc scenario. The secondary user network structure is based on beamforming at both the transmitter ( $K$ antennas) and the receiver ( $K$ antennas) for each secondary user link. The transmission scheme is characterized by the power allocation, eigenvalues and eigenvectors of the transmit covariance matrix.

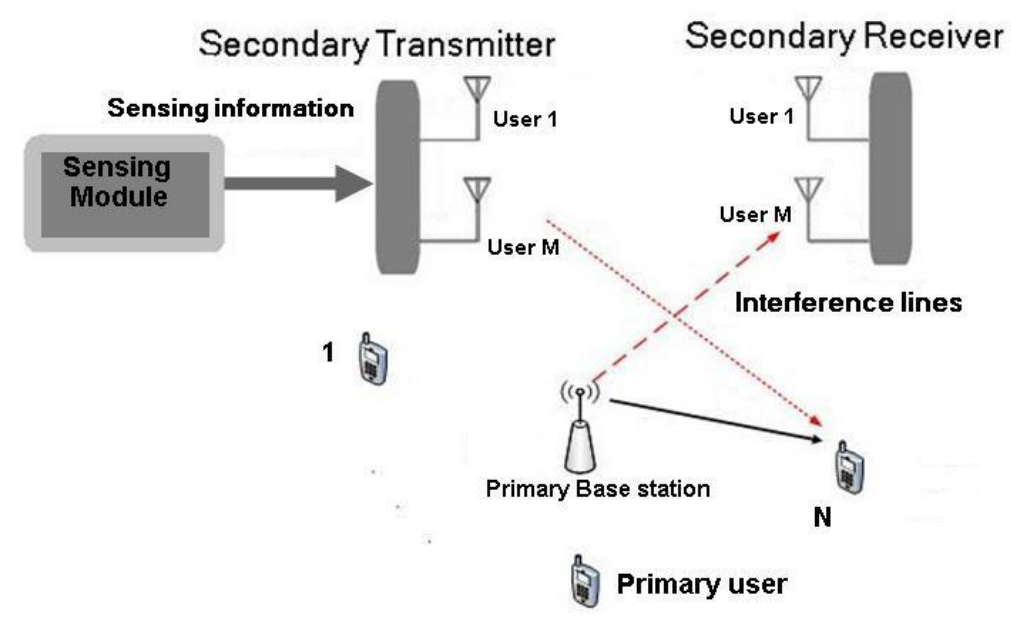

Fig1. Conceptual diagram of the system model

\section{Problem Formulation And Solution}

\subsection{Power Control in Underlying Mode}

The objective here is considered as to maximize the transmission capacity of the secondary users 
subject to minimum interference and maximum quality of service of the primary users with minimum transmission power for secondary users by beamforming approach. According to [8] the nth primary user's received signal is obtained as follow:

$$
\mathbf{y}_{\mathbf{n}}=\mathbf{h}_{p u_{n}} \cdot x_{p u}+\sum_{j=1}^{M} \mathbf{h}_{p u_{j}} \cdot \mathbf{b}_{j} \cdot x_{j}+\mathbf{n}_{m}
$$

Where $x_{p u}$ and $x_{j}$ are the transmitted signals of the primary base station and secondary users, respectively. The power of associated signals for $j=1,2,3 \ldots \ldots m$ are as:

$$
E\left[\left|\mathbf{n}_{m}\right|^{2}\right]=E\left[\mathbf{n}_{m} \mathbf{n}_{m}^{H}\right]=N_{0}^{2}, E\left[\left|x_{j}\right|^{2}\right]=p_{s u} \text { and } E\left[\left|x_{p u}\right|^{2}\right]=p_{p u}
$$

$\mathbf{h}_{p u}$ and $\mathbf{H}_{s u}$ are vectors in size of $\mathrm{k} \times 1$ and $\mathrm{k} \times \mathrm{k}$, the fading path gains from primary base station to the nth primary users and channel gains between secondary users, respectively. ${ }^{m}$ denotes zero mean additive with Gaussian noise with variance $N_{0}^{2}$. Define $p_{p u}$ and $p_{s u}$ as the transmitted power of primary base station and secondary users, respectively. Also power $p_{s u}$ is constrained by a maximum transmit power limit $p_{\max }$. We design the transmit and receive beamvectors as we mentioned in [8] then we can express the mth secondary user received signal as:

$$
\mathbf{y}_{\mathbf{m}}=\mathbf{H}_{s u m m} \cdot \mathbf{s}_{m}+\sum_{j=1, j \neq m}^{M} \mathbf{H}_{s u j m} \cdot \mathbf{s}_{j}+\mathbf{h}_{p u_{m}} \cdot x_{p u}+\mathbf{n}_{m}
$$

The transmit vector of size $\mathrm{K} \times 1$ is $\mathbf{S}_{m}=\mathbf{b}_{m} \cdot x_{m}$, Where $\mathbf{b}_{m}$ is the pre-beamforming vector and $x_{m}$ is the transmit sample for $\mathrm{m}$ between 1 and $M$. Where $\mathbf{a}_{m}$ is the post-beamforming vector at the receive secondary users. The signal to interference noise ratio at the mth secondary user is as follow according to [8].

$$
\operatorname{SINR}_{s u}=\frac{E\left[\left|\mathbf{a}_{m}^{H} \cdot \mathbf{H}_{s u_{m m}} \cdot \mathbf{b}_{m} \cdot x_{m}\right|^{2}\right]}{E\left[\left|\mathbf{a}_{m}^{H} \cdot \sum_{j=1, j \neq m}^{M} \mathbf{H}_{s u_{m j}} \cdot \mathbf{b}_{j} \cdot x_{j}+\mathbf{a}_{m}^{H} \cdot \mathbf{h}_{p u_{m}} \cdot x_{p u}+\mathbf{a}_{m}^{H} \cdot \mathbf{n}_{m}\right|^{2}\right]}
$$

The per-user sum capacity is:

$$
C_{s u}=\sum_{m=1}^{M} \log _{2}\left(1+\frac{\left|\mathbf{a}_{m}^{H} \cdot \mathbf{H}_{s u_{m m}} \cdot \mathbf{b}_{m}\right|^{2} \cdot p_{s u}}{\sum_{j=1, j \neq m}^{M}\left|\mathbf{a}_{m}^{H} \mathbf{H}_{s u_{m j}} \cdot \mathbf{b}_{j}\right|^{2} \cdot p_{j}+\left|\mathbf{a}_{m}^{H} \cdot \mathbf{h}_{p u_{m}}\right|^{2} \cdot p_{p u}+\left|\mathbf{a}_{m}^{H}\right|^{2} \cdot N_{0}^{2}}\right)
$$

The maximum eigenvalue of $\mathbf{H}_{s u_{m m}}^{H} \cdot \boldsymbol{\Phi}_{s u}^{-1} \cdot \mathbf{H}_{s u_{m m}}$ is defined as $\lambda_{\max }(m)$ and must be chosen to maximize the capacity of secondary users so: 
$C_{s u}=\sum_{m=1}^{M} \log _{2}\left(1+\lambda_{\max }(m) \cdot \mathbf{b}_{m}^{H} \cdot \mathbf{b}_{m}\right)$

Subject to: $\sum_{j=1}^{M} \mathbf{b}_{j}^{H} \cdot \mathbf{b}_{j}=\sum_{j=1}^{M}\left\|\mathbf{b}_{j}\right\|^{2} \leq M \cdot p_{\max }$

$\left\|\mathbf{b}_{m}\right\|^{2} \leq p_{\max }$ for $\quad m=1,2, \ldots . M$

$E\left[\left|\mathbf{h}_{p u_{j}} \mathbf{b}_{j}\right|^{2}\right]=\left(\mathbf{h}_{p u_{j}} \mathbf{b}_{j} \cdot \mathbf{b}_{j}^{H} \cdot \mathbf{h}_{p u_{j}}^{H}\right)=\left\|\mathbf{h}_{p u_{j}} \cdot \mathbf{b}_{j}\right\|^{2} \leq Q_{\mathrm{int}}$

For beamforming, the transmitted power through all the secondary users for the mth secondary user is proportional to $\left\|\mathbf{b}_{m}\right\|^{2}$. We can convert the constrained optimization process into an unconstrained one to meet problem constraints simultaneously. The behavior of cost function is dynamic due to nonstationary environment specifications. The Lagrangian cost function becomes as below we should minimize it as fitness function:

Min $\left.L=-\sum_{m=1}^{M} \log _{2}\left(1+\lambda_{\max }(m) \cdot \mathbf{b}_{m}^{H} \cdot \mathbf{b}_{m}\right)+\alpha_{1} \cdot\left(\sum_{j=1}^{M}\left\|\mathbf{b}_{j}\right\|^{2}\right)-M \cdot p_{\max }\right)+$

$\alpha_{2} \cdot\left(\left\|\mathbf{b}_{j}\right\|^{2}-p_{\max }\right)+\alpha_{3} \cdot\left(\left\|\mathbf{h}_{p u_{j}} \cdot \mathbf{b}_{j}\right\|^{2}-Q_{\text {int }}\right)$

Where $Q_{\text {int }}$ is the maximum tolerable received power at the primary receiver and $\alpha_{i}=\left\{\alpha_{1}, \alpha_{2}, \alpha_{3}\right\}$ is the Lagrangian multiplier. To achieve the optimal performance, Lagrangian multipliers, pre and post beam forming vectors are adjusted to let the transmit power satisfy all constraints by PSO[10].

\subsection{Spectrum Sensing in Overlay Mode}

Energy detector based approach, also known as radiometry or periodogram is the most common way of spectrum sensing because of its low computational and implementation complexities. Also, receivers do not need any knowledge on the primary user's signal. The method has also been analyzed in the presence of signals with random amplitude and channel fading. For a low pass signal having bandwidth $W$, the energy in a sample record of duration $T$ is approximated by $2 T W$ where the received waveform is sampled at the rate $2 W$. The energy is expressed as follow:

$$
E=\int_{0}^{T} n^{2}(t) d t \approx \frac{1}{(2 W)} \sum_{i=1}^{2 T W} a_{i}^{2}
$$

Where $n(t)$ is Gaussian noise process and $\alpha_{i}$ is the ith noise sample the results are the same for a band pass processes provided that $W$ is interpreted as the positive frequency bandwidth. For zero mean Gaussian distributed noise only $\left(H_{0}\right)$, the energy $E$ follows central chi-square $\left(\chi^{2}\right)$ distribution with $2 T W$ degrees of freedom. In the case that the primary user/ signal is present $\left(H_{1}\right)$, $E$ follows a non-central chi-square $\left(\chi^{2}\right)$ distribution with $2 T W$ degrees freedom and a noncentrality parameter $2 \gamma$, where $\gamma$ is the detected signal- to- noise-ratio (SNR). 


$$
E=\left\{\begin{array}{cc}
\chi_{2 T W}^{2} & H_{0} \\
\chi_{2 T W}^{2}(2 \gamma) & H_{1}
\end{array}\right.
$$

The probability of false alarm $p_{f}$ and the probability of correct detection $p_{d}$ are described as:

$$
\begin{aligned}
& \left.p_{f}=p_{r}(E\rangle \xi \mid H_{0}\right)=\frac{\Gamma\left(\tau_{b}, \frac{\zeta}{2}\right)}{\Gamma\left(\tau_{b}\right)} \\
& \left.p_{d}=p_{r}(E\rangle \xi \mid H_{1}\right)=Q_{\tau_{b}}(\sqrt{2 \gamma}, \sqrt{\zeta})
\end{aligned}
$$

The joint probability for detection $Q_{d}$ and false alarm $Q_{f}$ can therefore be given as:

$$
\begin{aligned}
& Q_{d}=1-\left(1-P_{d}\right)^{n} \\
& Q_{f}=1-\left(1-P_{f}\right)^{n}
\end{aligned}
$$

Where $\mathrm{n}$ denotes number of secondary users and $p_{d}$ and $p_{f}$ denote probability of detection and false alarm. Where $\zeta$ is the decision threshold, $\Gamma$ is the gamma function, both complete and incomplete types are used, and $\tau_{b}=T W$ is the time bandwidth and $Q_{\tau_{b}}$ is the Marcum $Q$-function [9]. When spectrum holes have been detected the transmission power of secondary users can be maximized for maximum capacity so the inequality equation can convert to equality as follow:

$$
\begin{aligned}
& \sum_{j=1}^{M} \mathbf{b}_{j} \cdot \mathbf{b}_{j}^{H}=\sum_{j=1}^{M}\left\|\mathbf{b}_{j}\right\|^{2}=M \cdot p_{\max } \\
& \left\|\mathbf{b}_{m}\right\|^{2}=p_{\max }, \text { for } \quad m=1,2, \ldots . M
\end{aligned}
$$

\section{NUMERICAL RESUlTS}

In simulation model, the problem of resource allocation in the context of secondary radio networks has been simulated. The combination of spectral detection with evolution optimization in the cognitive network and trying to increase the system output rate has been used.With the deployment of $K$ antennas at the secondary transceivers, an efficient transmit beamforming technique is proposed. In our PSO algorithm the three Lagrange multipliers will be set during each iteration to their best values. Path noises are independent zero-mean complex Gaussian random variable with variance1. The maximum transmit power for secondary users are assumed to be $5 \mathrm{~mW}$. Interference from primary users to base station is ignored. Interference constraint of all primary users is $10^{-4}$. Sensing time of 2 micro second is considered. We use $T=40$ and the number of users is 4,8 and 15 and making four decision thresholds in simulations. It was found that the more repetition of the algorithm in each iteration has the much accuracy. In figure 2 the probability of detection and false detection are shown. It can be seen from the figure 2-(a) that by increasing the number of cooperative users, the probability of false alarm is reduced. From figure 2-(b), it can be seen that with increasing the number of users the probability of detection arises. 


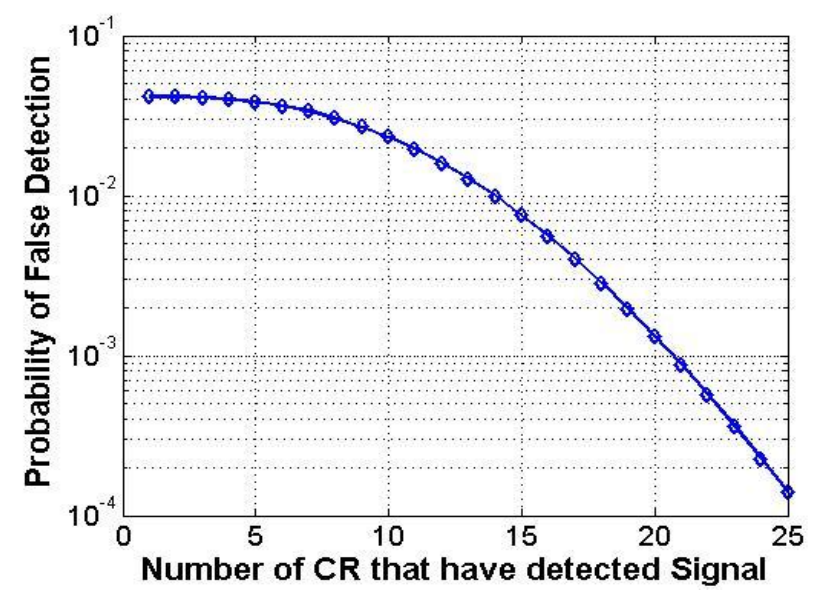

(a)

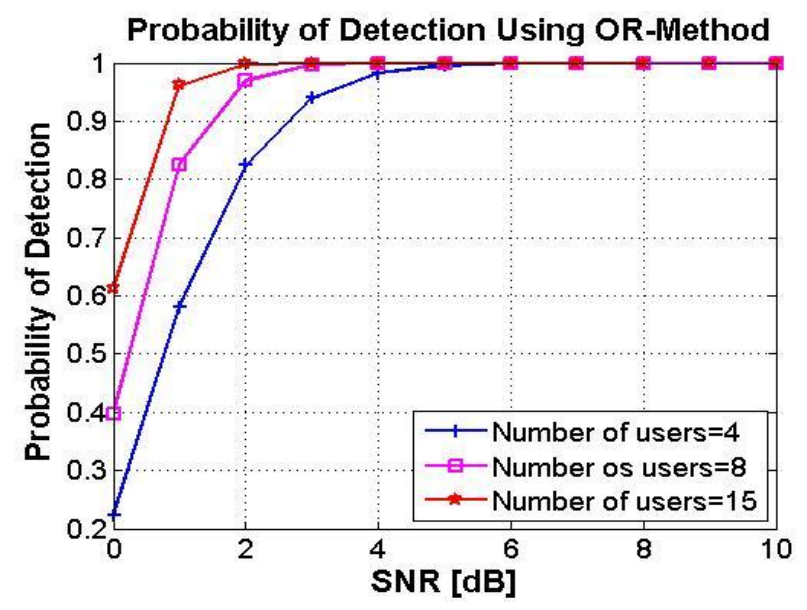

(b)

Fig.2. (a) Probability of false detection, (b) Probability of detection

By making average global information of the best particles, the accuracy of the best particles has been raised. We can see the behavior of the cost function and its convergence attributes of PSO, it is clear that the all constraints are fulfilled. From figure 3-(a), it can be seen that the transmission capacity arises with increasing the amount of transmission power; however the power will be limited by our simulations constraints and primary user interference. Figure 3-(b) shows allocated transmission power of secondary users. The fitness functions steer the evolution of the PSO in the correct direction to optimize the given multi-objective functions for the secondary users with the defined constraints.

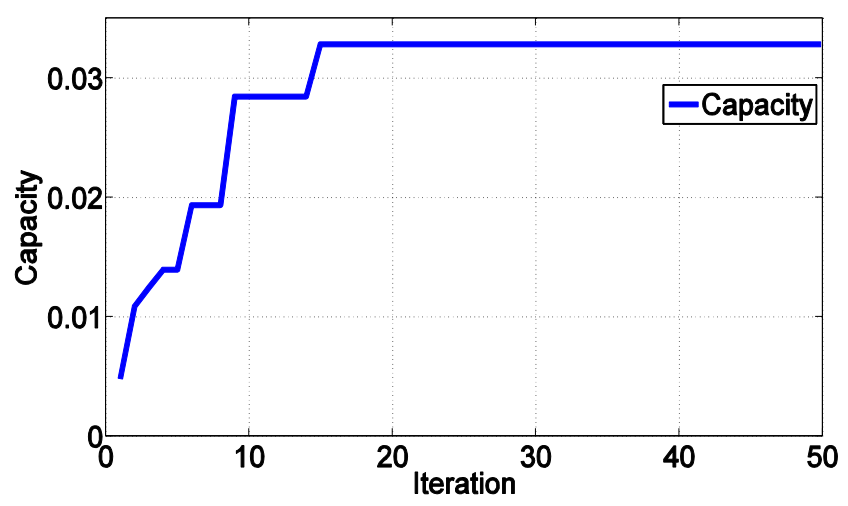

(a) 


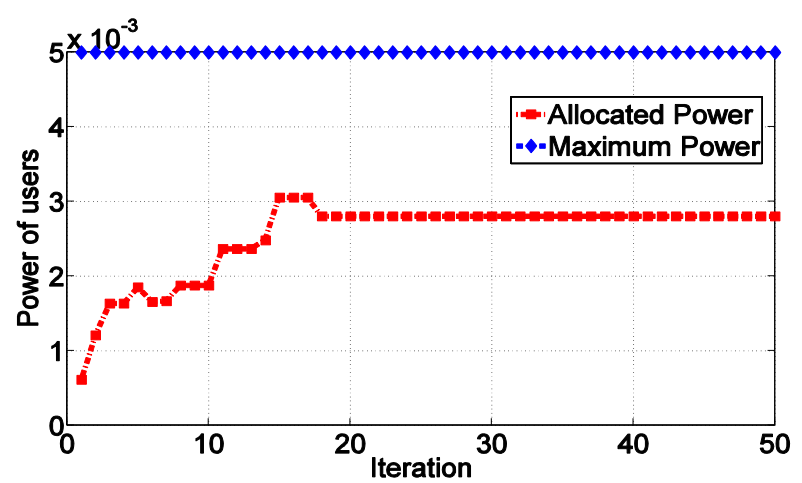

(b)

Fig3. (a) Transmission capacity of the secondary users, (b) Convergence of transmission power for secondary users

With this combination of sensor system and spectrum detection and concurrency detection, the efficiency of the higher model went up and improved.

\section{CONCLUSION}

We have proposed a PSO assisted scheme to design of distributed power control by beam forming in secondary radio networks. In addition, spectrum sensing or awareness has been investigated. The minimum transmission power of each secondary or secondary user is considered, when primary users are in active mode and frequency spectrum has been occupied. Proposed scheme shows the performance of a heuristic improvement in secondary radio performance. Reliable detection of the presence of primary users is of uttermost importance since the secondary radio operating, as a secondary system is not allowed to cause harmful interference to the primary user. We have evaluated the performance of radiometry in terms of the probabilities of detection and false alarm. Due to the limited awareness of a single secondary radio node, cooperative sensing will be important in practical secondary systems. This would lead to a tradeoff between reliable sensing information and the costs causes by more extensive cooperation, such as complexity and increased signaling. Spectral radiography in our system, especially in non-stationary model, has been played a great role in early detection of interferences.

\section{REFERENCES}

[1] A. Ghasemi and E. S. Sousa, Spectrum sensing in cognitive radio networks: Requirements, challenges and design trade-offs, IEEE Comm. Mag., vol. 46,pp. 32-39, Apr. 2008.

[2] D. Čabrić and R. W. Brodersen, "Robust Spectrum Sensing Techniques for Cognitive Radio Networks," in Towards Cognitive and Cooperative Wireless Networking: Techniques, Methodologies and Prospects edited by F. Fitzek and M. Katz, pp. 373-394, Springer, 2007.

[3] S. Grandhi, J. Zander, R. Yates, “Constrained Power Control," Int. J. of Wireless Personal Commun., Vol. 1, no. 4, Apr. 1995.

[4] T. Renk, C. kloeck, D. Burgkhardt, FK. Jondral, D. Grandblaise, S. Gault, JC. Dunat, "Bio-inspired algorithms for dynamic resource allocation in cognitive wireless networks," Mobile Netw. Appl., pp. 431$441,2008$.

[5] Z. Zhao, S. Xu, S. Zheng, J. Shang, "Cognitive radio adaptation using particle swarm optimization,” Wiley J. on Wireless Commun. and Mobile Comp., 2008.

[6] MH. Islam, YC. Liang, AT. Hoang, "Joint power control and beamforming for cognitive radio networks," In IEEE Trans. On Wireless Commun., Vol. 7, no. 7. Jul. 2008. 
[7] P. Derakhshan B., G. Dadashzadeh, F. Razzazi, S. M. Razavizadeh, "Minimum power transmission design for cognitive radio networks in non-stationary environment," IEICE Electronic Exp., Vol. 8, no. 3, pp.136-142, Feb. 2011.

[8] P. Derakhshan, G.R. Dadashzadeh, F. Razzazi, S.M. Razavizadeh, "Bio-inspired distributed beamforming for cognitive radio networks in non-stationary environment.” IEICE Electronic Exp. vol. 8, no. 6, 2011.

[9] P. Derakhshan, "Modified Spectrum Sensing and Awareness in Wireless Radio Networks," Int. Review on Modeling and Simulations, I.RE.MO.S. Journal, pp. 718-722, Vol. 4. No. 2, 2011.

[10] P. Derakhshan, G. Dadashzadeh, F. Razzazi,S. M. Razavizadeh, "Power and Time Slot Allocation in Cognitive Relay Networks Using Particle Swarm Optimization,” Hindawi Journal, 2013.

Citation: Pouya Derakhshan-Barjoei. (2017). Joint Resource Allocation and Spectral Radiometry in NonStationary Cognitive Radio Networks, International Journal of Innovative Research in Electronics and Communications (IJIREC), 4(2), pp.18-25, DOI: http:// dx.doi.org/10.20431/2349-4050.0402004

Copyright: (C) 2017 Pouya Derakhshan-Barjoei. This is an open-access article distributed under the terms of the Creative Commons Attribution License, which permits unrestricted use, distribution, and reproduction in any medium, provided the original author and source are credited 